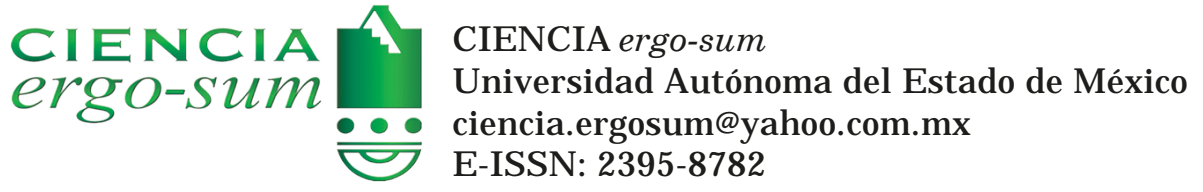

\title{
Modos de vida rurales en el Área de Protección de Flora y Fauna Nevado de Toluca
}

\author{
Ruiz Torres, Mónica Elizama
}

Modos de vida rurales en el Área de Protección de F lora y Fauna N evado de Toluca

CIENCIA ergo-sum, vol. 26, núm. 1, marzo-junio 2019| e40

Universidad Autónoma del Estado de México, México

Esta obra está bajo una Licencia Creative Commons Atribución-NoComercial-SinDerivar 4.0 Internacional.

Ruiz Torres, M. E. (2018). Modos de vida rurales en el Área de Protección de Flora y Fauna Nevado de Toluca. CIE NCI A ergo-sum, 26 (1). https://doi.org/10.30878/ces.v26n1a5 


\title{
Modos de vida rurales en el Área de Protección de Flora y Fauna Nevado de Toluca
}

Rural Livelihoods in the Nevado of Toluca Wildlife Protection Area

Mónica Elizama Ruiz Torres

Universidad Autónoma de San Luis Potosí, México

monica.ruiz.torres24@gmail.com

Recepción: 22 de agosto de 2017

Aprobación: 12 de febrero de 2018

\section{RESUMEN}

Se caracterizan los modos de vida rurales desarrollados por los pobladores de Raíces, Zinacantepec, que se ubica dentro del perímetro del área de protección de flora y fauna Nevado de Toluca (APNT). Los datos mostrados fueron obtenidos a partir del uso de métodos cualitativos, de donde se analizaron 29 grupos domésticos seleccionados a partir de un muestreo no probabilístico. Se detectaron tres modos de vida en la región: modo de vida agrícola, del empleado en el sector servicios y jornalero agrícola. En conclusión, se requiere que el Estado desarrolle un plan de manejo integral que considere los tres modos de vida rurales detectados en el APNT, puesto que en el actual programa de manejo no se contemplan.

Palabras Clave: estrategias de subsistencia, modos de vida rurales, sistemas agrícolas, grupos domésticos.

\begin{abstract}
:
The aim of the research was to characterize the rural livelihoods developed by people from Raíces, Zinacantepec; who are located in the perimeter of the Protected Area of Flora and Fauna Nevado de Toluca (APNT). Data were collected through qualitative methods from 29 domestic groups, which were selected with non-probabilistic sampling method. Three rural livelihoods were identified in the region such as: agricultural livelihood, employee sector services and agricultural day labourer. It is concluded that government policies require a holistic management plan that consider the three rural livelihoods identified in the APNT, since these are not considered into the current policy plan of management.
\end{abstract}

KEYWORDS: subsistence strategies, rural livelihoods, agricultural systems, households.

\section{INTRODUCCIÓN}

En casi toda América Latina la tendencia de creación en materia legal de áreas naturales protegidas (ANP) presupone un discurso conservacionista que difícilmente puede ser aplicado de manera total, puesto que los asentamientos humanos en parques nacionales son una realidad. En México viven alrededor de 2591147 habitantes en zonas consideradas áreas naturales protegidas; esto representa $2.5 \%$ de la población total del país y 5.7\% de la población rural total (Conanp, 2016), lo que presupone un reto para la conservación. Sin embargo, al ser un país miembro de la Organización de las Naciones Unidas (ONU), se ha unido a las políticas conservacionistas que este organismo impulsa en materia de sustentabilidad. Una prueba de ello es la Ley General del Equilibrio Ecológico y la Protección al Ambiente (2016), la cual define el desarrollo sustentable como el proceso evaluable mediante criterios e indicadores de carácter ambiental, económico y social que tiende a mejorar la calidad de vida y la productividad de las personas y está fundada en medidas apropiadas de preservación del equilibrio ecológico, protección del ambiente y aprovechamiento de recursos naturales, de manera que no se comprometa la satisfacción de las necesidades de las generaciones futuras. 
Bajo este esquema político-legal de sustentabilidad, en el 2000 surge la Comisión Nacional de Áreas Protegidas que, al desconcentrarse de la Secretaría del Medio Ambiente y Recursos Naturales (Semarnat), adquiere autoridad sobre la administración de todas las reservas mexicanas de la biósfera que actualmente suman 176, las cuales ocupan una superficie territorial de más de 25394379 hectáreas divididas en nueve regiones del país (Conanp, 2016).

El Área de Protección de Flora y Fauna Nevado de Toluca (APNT) pertenece a la región Centro y Eje Neo Volcánico. Además de formar parte del Sistema Volcánico Transversal, El Nevado de Toluca es el cuarto volcán más grande de México, formado por sucesivas capas de lava y un cráter de forma elíptica (Conanp, 2016). Cabe destacar que ocupa porciones de 11 municipios del Estado de México, con una extensión territorial total de 53590 hectáreas de ecosistemas forestales y una población estimada de 5397 habitantes (Conanp, 2016). En el Valle de Toluca es la fuente principal de servicios ambientales tales como la calidad del aire y agua, protección de la biodiversidad, provisión de biomasa y captación de agua.

Con estos antecedentes, el objetivo de este trabajo fue caracterizar los modos de vida rurales desarrollados por los pobladores de Raíces, municipio de Zinacantepec, localidad ubicada dentro del perímetro del Área de Protección de Flora y Fauna Nevado de Toluca (APNT).

\section{Metodología}

Los datos mostrados se obtuvieron a partir del empleo de métodos cualitativos, y se puso especial énfasis en las características de los sistemas de producción. La recopilación de datos inició con la demarcación del área de estudio y con los recorridos de área para el reconocimiento de la zona. El escenario seleccionado fue el Área de Protección de Flora y Fauna Nacional Nevado de Toluca (APNT), en específico la delegación de Raíces, municipio de Zinacantepec, ubicada al suroeste de Toluca, Estado de México, en las coordenadas geográficas $99^{\circ} 48^{\prime} 14^{\prime \prime}$ de longitud este y 1909'37” de latitud norte a unos 3500 metros sobre el nivel del mar (INEGI, 2010).

Dichos recorridos buscaron recabar información sobre las características sociales y culturales de los modos de vida en la zona, así como del manejo de los recursos naturales. Para fines de esta investigación, se analizaron 29 grupos domésticos que representan $20.56 \%$ del total de la zona de estudio. La muestra fue seleccionada a través de un muestreo no probabilístico o bola de nieve y se buscó a aquellos productores que en el contexto tuvieran mayores extensiones de terreno, cosechas y cabezas de ganado, así como quienes en contraposición tuvieran lo mínimo en todas las categorías.

Se realizó observación directa y participante con los grupos domésticos poniendo énfasis en las actividades productivas y la distribución de tareas de su vida cotidiana. Adicionalmente, se aplicaron cuestionarios a todos los jefes de familia con el objetivo de determinar las actividades productivas principales, manejo de recursos forestales, organización social y roles domésticos. Se aplicó también una entrevista estructurada a tres informantes clave: dos a Delegados Municipales y una al Comisario Ejidal. Adicional, se aplicó una entrevista no estructurada al Fiscal, una autoridad religiosa. Cabe mencionar que por cuestiones éticas se omitieron los nombres de los entrevistados.

La delegación municipal de Raíces posee el régimen de propiedad conocido como Ejido. Esta delegación pertenece al Ejido de Loma Alta, constituido por un total de 1272 hectáreas (Abasolo Patricio, 2006). En el aspecto demográfico, su población total es de 664 habitantes, de los cuales 331 son hombres que representan $49.84 \%$ y 333 son mujeres que equivalen a 50.15\% respecto del total (INEGI, 2010). Estas personas constituyen un total de 141 hogares censales, de los cuales $93.61 \%$ tiene jefatura masculina y $6.38 \%$ jefatura femenina. Los hogares tienen en promedio 4.6 miembros de familia. De las viviendas habitadas, $90 \%$ tiene conexión de luz eléctrica, agua entubada y drenaje. El grado de marginación presente en la localidad según el Conapo (2010) es alto y ocupa el puesto 2415 en el contexto estatal. 
Desde hace poco más de cuarenta años, la actividad económica principal es la agricultura, de la cual resaltan los cultivos forrajeros de avena y maíz, así como el cultivo de papa. La segunda más importante es la cría y explotación de animales, en particular de ganado bovino. El grado promedio de escolaridad de los jefes de familia es de seis años concluidos, es decir, primaria terminada.

\section{Algunas consideraciones teóricas sobre el enfoQue de modos de Vida}

El enfoque conceptual de modos de vida surge a finales de la década de los noventa a causa de la necesidad de nuevos paradigmas teóricos que mostraran eficacia en el diseño de estrategias de combate a la pobreza. Se puso énfasis en las zonas rurales, pues se estimaba que $70 \%$ de la población mundial habitaba estas zonas bajo dicha condición (Carney, 1999). Para Chambers y Conway (1992) un modo de vida implica el medio para ganarse el sustento, el cual combina tres elementos: a) el primero, la capacidad, es decir, lo que una persona es apta de hacer, $b$ ) en segundo lugar, la equidad, que refiere a la distribución de la riqueza y los recursos tangibles y $c$ ) la sustentabilidad, que involucra el cuidado de la naturaleza y se relaciona directamente con el acceso a los recursos e incluye también nociones intangibles como leyes y normas.

Ellis (2000) retoma el concepto original de Chambers y Conway, pero enfatiza en la diversidad de actividades que los hogares realizan para sobrevivir y elevar su calidad de vida. Esta diversificación, según Farrington et al. (1999), posibilita reducir la vulnerabilidad de la población, ya que aparte de las labores agrícolas se busca un empleo asalariado (incluyendo la migración). Esta circunstancia permite a los grupos domésticos anticiparse y equilibrar las épocas de crisis.

Para Ellis (2000) este enfoque teórico retoma el análisis de cinco capitales como los activos de los modos de vida. a) El capital natural involucra los recursos naturales como la tierra, agua, árboles y todos aquellos derivados de la biodiversidad que son utilizados por la gente para obtener lo necesario para vivir. $b$ ) El capital físico representa el acceso a la producción económica mediante herramientas de trabajo y todo aquel objeto que es creado como los edificios, canales de irrigación, caminos y máquinas. c) El capital humano está representado por la mano de obra dada por los grupos domésticos influida directamente por el nivel educativo y la salud de la población. d) El capital social comprende las asociaciones y el trabajo colectivo que permite que los habitantes se relacionen entre sí. e) El capital financiero engloba el acceso al dinero como créditos, remesas y salarios, entre otros.

El estudio de los modos de vida ha permitido documentar una creciente diversificación de actividades productivas por las familias que viven en contextos rurales, así como de los roles domésticos y los derechos de acceso, uso y control de los activos que cada miembro de la familia posee.

\section{Modos DE VIDA EN EL APNT}

Los modos de vida en Raíces están basados en el uso de recursos naturales contenidos en el área de protección de flora y fauna. Se detectaron tres modos productivos en el área de estudio: a) modo de vida agrícola, $b$ ) modo de vida del empleado en el sector servicios y $c$ ) el modo de vida del jornalero agrícola.

\section{Modo de Vida Agrícola}

Este modo de vida incluye aquellos hogares que obtienen la satisfacción de sus necesidades básicas a través de actividades agrícolas. Se caracteriza por la posesión de terrenos ejidales destinados especialmente al cultivo de avena, papa y maíz. El rango de posesión de tierras va de 1 a 10.5 ha. Aunado a lo anterior, 36\% de los casos analizados tenía rentadas hasta 2 ha que fueron destinadas a la labor agrícola. 
En este modo de vida se detectaron tres subsistemas de producción:

a) Agrícola. Su fuente principal de ingresos es la agricultura de avena y maíz; sin embargo, puede haber presencia de hasta 20 cabezas de ganado (borregos).

b) Ganadero. La ganadería ovina representa su principal fuente de ingresos. El rango de cabezas va de 20 a 140 y se practica el pastoreo en el área de uso común del ejido. Estas actividades son complementadas con la agricultura, en especial de avena, papa y maíz, destinada para el autoconsumo, aunque de presentar excedente se comercializa en el mercado de la localidad y del municipio.

c) Trutícola. La actividad económica se centra en la crianza y venta de truchas. La granja de truchas está ubicada a cinco y medio kilómetros de distancia del asentamiento humano, ocupa una superficie de dos y media hectáreas y es de ciclo completo. El ciclo productivo de la granja dura un año, del cual se producen 350000 truchas; de esta producción, $71 \%$ se vende como alevines a productores de engorda. El porcentaje restante termina de crecer y se vende en diversos restaurantes de la zona y en el mercado del municipio. En cuanto a la agricultura, el cultivo principal es la avena.

Todos los grupos domésticos analizados hacen uso de mano de obra familiar, aunque se contratan jornaleros agrícolas en las épocas específicas de siembra y cosecha. Para Pierre Dupraz (2015) la mano de obra familiar y la contratada son complementarias y sólo menos de $1 \%$ de las fincas agrícolas no contrata jornaleros. PosadasDomínguez et al. (2014) consideran la mano de obra familiar como un elemento económico clave que tiene efectos directos sobre la competitividad de los sistemas agrícolas familiares. Evans et al. (2015) expresan que los jóvenes y niños contribuyen a la economía familiar con actividades agrícolas no remuneradasm, asociadas comúnmente con el trabajo femenino como el cuidado del ganado de traspatio.

En la zona de estudio, $18 \%$ de los agricultores cuenta con invernaderos de $250 \mathrm{~m}^{2}$ para la producción de semilla de papa. Cada estructura produce de setecientos cincuenta kilos a una tonelada de semilla con la posibilidad de obtener dos cosechas al año.

La ganadería de traspatio está presente como una estrategia de ahorro e intercambio en $100 \%$ de los grupos domésticos analizados dentro de esta categoría. Las especies que sobresalen son las aves de corral y los borregos. Para López González et al. (2012) la importancia de la ganadería de traspatio radica en que los productos obtenidos pueden ser destinados al autoconsumo y, en caso de necesidad económica, a la venta para asegurar la supervivencia de la familia. Stroebel et al. (2011) mencionan que el autoconsumo y venta del producto final, la acumulación de capital como "ahorro vivo" y el estatus social que genera la propiedad del ganado son las razones principales por las cuales los grupos domésticos conservan e incrementan la ganadería de traspatio.

Aunado a la ganadería de traspatio, el uso de otros recursos del bosque es una constante en este modo de vida; en este aspecto, sobresale la recolección de leña para autoconsumo, con una extracción promedio de tres y media toneladas cada seis meses y la recolección de hongos comestibles, tanto para autoconsumo como venta, de hasta veinte kilos por mes durante la temporada de lluvias. Para Llano (2014) este tipo de prácticas es reconocido como una importante estrategia en las prácticas de subsistencia de los grupos humanos. Kim et al. (2012) expresan que los recursos forestales pese a no ser clave en la economía de mercado sí son relevantes para la cultura y la economía familiar de los grupos domésticos, ya que se usan como comida, medicina, en rituales, materia prima para manufactura de ropa y artesanías, entre otros.

La forma de explotación del bosque constituye parte del conocimiento tradicional de los grupos domésticos sobre su medio geográfico. Este conocimiento se transfiere de una generación a otra a través de la observación y la práctica de los infantes, que comienza cuando el niño tiene entre 7 y 10 años y es capaz de acompañar al padre al bosque. Giday et al. (2016) ponen de manifiesto que en estos casos, donde convergen el conocimiento autóctono y la sostenibilidad de los recursos, la conservación de áreas naturales presenta su primer reto, pues hace necesario que el conocimiento tradicional y el cuidado de la biodiversidad se refuercen mutuamente. 


\section{Modo DE VIDA DEL EMPLEAdo EN EL SECTOR SERVICIOS}

Este modo de vida está caracterizado por la principal fuente de ingresos, que es el trabajo asalariado del jefe de familia. Destacan los trabajos de policía, chofer, servidores públicos, negocios particulares, entre otros. Las actividades agrícolas son complementarias y cada grupo doméstico posee entre una y siete hectáreas de terreno productivo; sin embargo, en promedio sólo siembran la mitad y el resto es rentado a ejidatarios de la zona. Los cultivos principales son papa, avena y maíz.

Para Ellis et al. (1999) la búsqueda de empleos asalariados fuera de los sistemas agrícolas implica poco o ningún riesgo de capital, de ahí que los sistemas familiares sean los primeros en buscar diversificar actividades. Sofer (2001) considera que la agricultura se ve beneficiada por estos ingresos extra, puesto que las familias invierten los recursos de los empleos no agrícolas en la modernización del equipo y otros activos. Si las comunidades se ubican en las proximidades de áreas metropolitanas, la pluriactividad puede generar un modo de vida alternativo delimitado por las condiciones geográficas y de mercado.

Para Vértiz (2016), debido a las bajas escalas productivas de los sistemas agrícolas latinoamericanos y los bajos índices de producción, en algunos casos es probable que los grupos domésticos no puedan prescindir de la combinación de actividades para mantenerse como productores agrícolas. Sraïri y Ghabiyel (2017) explican que el trabajo asalariado parece ser una variable de ajuste para mitigar los efectos de escasez de capital o tierra; además, en estos casos los productores suelen asignar más esfuerzos a la ganadería cuyos ingresos aseguran el mantenimiento de su modo de vida.

Coincidiendo con Sraïri y Ghabiyel (2017), la ganadería de traspatio en este modo de vida es de suma importancia. Los grupos domésticos poseen entre uno y diez borregos y hasta treinta aves de corral, que son vistas como una forma de acumulación de capital o simplemente como un "ahorro vivo" (Stroebel, 2011). Cabe mencionar que el pastoreo de los animales se realiza en las áreas de uso común del ejido y es realizado en particular por los niños y mujeres.

La participación femenina es básica para este modo de vida, en 100\% de los casos analizados la mujer era la responsable del cuidado de los animales de traspatio. Para Anthopoulou (2010) las mujeres son la verdadera mano de obra invisible en los sistemas mundiales de producción. En este aspecto, Rahman (2010) menciona que las mujeres contribuyen con cerca de $28 \%$ de las labores productivas y en ciertos casos incluso pueden sustituir las labores masculinas.

Para el caso específico de este modo de vida, tanto la ganadería de traspatio como la extracción de recursos del bosque (recolección de hasta tres y media toneladas de leña cada seis meses y de tres kilos de hongos comestibles cada semana) corresponden a actividades femeninas en $85 \%$ de los casos analizados.

\section{Modo de VIDA DEL JORnalero AGRíCola}

Los jornaleros agrícolas son personas dedicadas a la agricultura, pero, a diferencia de aquellos que se encuentran en el modo de vida agrícola, no tienen la posesión de los terrenos que trabajan sino que se emplean y reciben un sueldo por jornada que no necesariamente es otorgado por agricultores de la zona, dado que buscan contratarse en los ejidos aledaños o en trabajos agrícolas temporales de la región. Cabe mencionar que estos productores se autodefinen y comparten identidades como campesinos. Wahlhütter et al. (2016) refieren el término identidad a la singularidad y carácter personal que distingue a un grupo del resto, incluye cualidades de igualdad sobre la base del algún rasgo común conocido, que para el caso específico de este modo de vida se refiere a la labor agropecuaria.

Steward et al. (2001) consideran que al compartir un "estilo de vida" se moldean las identidades de grupo permitiendo posicionar al individuo como un tipo particular de sujeto, similar a otros dentro de la comunidad en algunos aspectos cruciales y diferentes de los excluidos. 
Cabe agregar que la recolección de recursos del bosque como hongos comestibles, hasta veinte kilos cada tercer día, y leña recolectada, a razón de tres y media toneladas cada dos meses, son actividades que refuerzan la identidad campesina y reproducen el sistema de generación en generación. Este traspaso generacional del conocimiento inicia de forma verbal y se refuerza con la práctica y la observación cuando el infante tiene entre 7 y 10 años y es capaz de acompañar a los padres al bosque.

\section{ConcLusión}

Pese a las recomendaciones por parte de las autoridades a limitar las actividades agropecuarias y de extracción forestal, existen al menos veinticinco comunidades similares a Raíces dentro del perímetro del Área de Protección de Flora y Fauna Nevado de Toluca (APNT), cuyos modos de vida rurales se centran en la herramienta básica del campesino: la tierra. Se requiere que el Estado desarrolle un plan de manejo integral que considere los tres modos de vida rurales detectados en la zona: $a$ ) agrícola, $b$ ) empleado en el sector servicios y $c$ ) jornalero agrícola, puesto que en el actual programa de manejo no se contemplan.

La ganadería de traspatio y la recolección de recursos forestales son una constante en los tres modos de vida rurales detectados en la región, por lo que son factores de conformación de identidades campesinas en el área estudiada.

La diversificación de actividades asalariadas es una estrategia de subsistencia entre los pobladores del APNT, la cual es visible notablemente en el modo de vida del empleado en el sector servicios y, en estos casos, la mujer es la responsable directa de transferir los sentimientos de identidad campesina a los hijos a través del traspatio.

Hoy en día hay mucho camino por recorrer en aras de conservar los recursos bióticos; por este motivo, las metodologías de investigación deben promover el análisis de la cultura en conjunto con el estudio del ambiente, puesto que aquí se podría encontrar el motor para el desarrollo que requiere de una reforma integral tripartita: a) en lo legal, $b$ ) en lo social (los modos de vida) y $c$ ) en los aspectos ecológicos. Por lo tanto, el éxito de los programas de combate a la pobreza dependerá de científicos sociales capaces de ampliar sus horizontes teóricos para trabajar de forma interdisciplinaria, ya que en la actualidad el reto implica enfrentarse a una realidad cada vez más compleja que demanda de la participación en conjunto de diversos especialistas tanto en las ciencias humanas como en las naturales.

\section{Prospectiva}

Para Herrera Tapia y Suárez Rincón (2015) el concepto de lo local es fundamental para el diseño de políticas públicas en poblaciones rurales asentadas en un territorio con características específicas propias. En este sentido, los resultados de esta investigación aportan la evidencia empírica necesaria para el diseño y adaptación de políticas públicas. El reto es lograr que los programas o cualquier otro instrumento de política combine los modos de vida de los pobladores en el APNT con los criterios de conservación dados por las instituciones oficiales como la Conanp (Comisión Nacional de Áreas Naturales Protegidas).

\section{Referencias}

Abasolo Palacio, V. (2006). Entre el cielo y la tierra: Raíces un pueblo de la alta montaña en el Estado de México. México: Universidad Iberoamericana.

Anthopoulou, T. (2010). Rural women in local agrofood production: Between entrepreneurial iniciatives and families strategies. A case study in Greece. Journal of Rural Studies, 26, 394-403. 
Carney, D. (1999). Approaches to sustainable livelihoods for the rural poor. Londres: Overseas Development Institute.

Chambers, R. Conway, G. (1992), Sustainable rural livelihoods: Practical concepts for the 21st Century. IDS Discussion Paper 296. Brighton: Institute of Development Studies.

Conanp (Comisión Nacional de Áreas Naturales Protegidas) (2016). Áreas Naturales Protegidas de México. Disponible en http://www.conanp.gob.mx/regionales/. Consultado el 11 de octubre de 2016.

Conapo (Consejo Nacional de la Población) (2010). Índice de marginación por localidad. CONAPO-Secretaría de Gobernación. Disponible en http://www.conapo.gob.mx/ES/CONAPO/Indice_de_Marginacion_ por_Localidad_2010. Consultado el 29 de septiembre de 2016.

Ellis, F. (2000). Rural livelihoods and diversity in developing countries. USA: Oxford University Press.

Ellis, N. E., Heal, O. W., Dent, J. B. y Firbank, L. G (1999). Pluriactivity, farm household socio-economics and the botanical characteristics of grass fields in the Grampian region of Scotland. Agriculture, Ecosystems \& Environment, 76, 121-134.

Farrington, J., Carney, D., Ashley, C. y Turton, C. (1999). Sustainable livelihoods in practice. Early applications of concepts in rural areas. Natural Resources Perspectives, 42, Londres: Overseas Development Institute.

Giday, K., Lenaerts, L., Gebrehiwot, K., Yirga, G., Verbist, B. y Muys, B. (2016). Ethnobotanical study of medicinal plants from degraded dry Afromontane forest in northern Ethiopia: species, uses and conservation challenges. Journal of Herbal Medicine, 6, 96-104.

Herrera Tapia, F. y Suárez Rincón, J. (2015). Agroindustria rural, proximidad y políticas públicas para el desarrollo territorial rural, en M. C. Hernández Moreno et al.(Ed.) Una revisión teórica. Territorio y gestión del desarrollo. Epistemologías y experiencias (pp. 209-232). México: Ediciones Plaza y Valdés-Conacyt.

INEGI (Instituto Nacional de Estadística y Geografía). (2010). Censo de Población y Vivienda 2010. Instituto Nacional de Estadística y Geografía. Disponible en http://www.inegi.org.mx/est/contenidos/proyectos/ ccpv/cpv2010/. Consultado el 29 de septiembre de 2016.

Kim, I. A., Trosper, R. L. y Mohs, G. (2012). Cultural uses of non-timber forest products among the Sts'ailes, British Columbia, Canada. Forest Policy and Economics, 22, 40-46.

Ley General del Equilibrio Ecológico y la Protección al Ambiente.(2016). Disponible en http://www.diputados. gob.mx/LeyesBiblio/pdf/148_130516.pdf. Consultado el 11 de octubre de 2016.

Evans, R., Mariwah, S. y Antwi, K. (2015). Struggles over family land? Tree crops, land and labour in Ghana's Brong-Ahafo region. Geoforum, 67, 24-35.

López González, J., Damián Huato, M., Álvarez Gaxiola, F., Parra Inzunza, F. y Zuluaga Sánchez, G. (2012). La economía de traspatio como estrategia de supervivencia en San Nicolás de los Ranchos, Puebla, México. Revista de Geografía Agricola, 48-49, 51-63.

Llano, C. (2014). La explotación de los recursos vegetales en sociedades cazadoras-recolectoras del sur de Mendoza, Argentina. Darwiniana. Nueva Serie, 2, 96-111.

Pierre Dupraz, L. (2015). Trends in family labour, hired labour and contract work on French field crop farms: The role of the Common Agricultural Policy. Food Policy, 51, 104-118.

Posadas-Domínguez, R., Arriaga-Jordán, C. y Martínez-Castañeda, E. (2014). Contribution on family labour to the profitability and competitiveness of small-scale dairy production systems in central Mexico. Tropical Animal Health and Production, 46, 235-240.

Rahman, S. (2010). Women's labour contribution to productivity and efficiency in agriculture: Empirical evidence from Bangladesh. Journal of Agricultural Economics, 6(2), 318-342. 
Sraïri, M. T. y Ghabiyel, Y. (2017). Coping with the work constraints in crop-livestock farming systems. Annals of Agriculture Science. Disponible en https://doi.org/10.1016/j.aoas.2017.01.001.

Sofer, M. (2001). Pluriactivity in the Moshav: family farming in Israel. Journal of Rural Studies, 17, 363-375.

Steward, R., Bechhofer, F., McCrone, D. y Kiely, R. (2001). Keepers of the land: Ideology and identities in the Scottish rural elite. Identities, 8(3), 381-409.

Stroebel, A., Swanepoel, F. J. C. y Pell, A. N. (2011). Sustainable smallholder livestock systems: A case study of Limpopo Province, South Africa. Livestock Science, 139, 186-190.

Vértiz, P. (2016). El rol de la pluriactividad en la persistencia de la producción familiar láctea en la Cuenca de Abasto Sur de Buenos Aires. Trabajo y Sociedad, 27, 475-499.

Wahlhütter, S., Vogl, C. R. y Eberhart, H. (2016). Soil as a key criteria in the construction of farmer's identities: The example of farming in the Austrian province of Burgerland. Geoderma, 269, 39-53.

CC BY-NC-ND 